\title{
A PARALLEL CONDENSATION-BASED METHOD FOR THE STRUCTURAL DYNAMIC REANALYSIS PROBLEM
}

\author{
ANDREAS PELZER AND HEINRICH VOSS \\ Technical University of Hamburg - Harburg, Section of Mathematics, D - 21071 \\ Hamburg, Germany, E-mail: \{pelzer,voss\}@tu-harburg.de
}

\begin{abstract}
In the dynamic analysis of a structure quite often a small number of the smallest eigenvalues and eigenmodes of a large and sparse general eigenvalue problem have to be determined assuming that good approximations to the demanded eigenmodes are available from previous computations. In this situation powerful approaches like Lanczos method or Jacobi-Davidson may be inferior to methods which in general are known to be slower. In this note we demonstrate this effect comparing P_ARPACK, the parallel version of ARPACK, with a parallel condensation method with general masters for a finite element model of a container ship.
\end{abstract}

\section{Introduction}

The response of a structure to dynamic excitations depends, to a large extent, on the natural frequencies of the structure. Excessive vibration occurs when the frequency of the excitation is close to one of the natural frequencies of the structure. Hence, in a design process one has to modify a structure several times to shift the natural frequencies of the structure out of the range of excitation frequencies. Typically, in each design step the structure is perturbed only by a small amount, and the modal shapes are not altered very much whereas the natural frequencies can change significantly. Therefore, in the design process reasonable approximations of the desired mode shapes are available from previous computations. A similar situation occurs in the dynamic analysis of a structure where the dynamic behaviour has to be determined for different loadings.

Powerful approaches like Lanczos or Jacobi-Davidson methods for sparse general eigenvalue problems

$$
K x=\lambda M x
$$

are not able to exploit the knowledge of good initial approximations to the set of desired eigenvectors, but they have to solve each eigenvalue problem from scratch. Hence, with a good approximation already available methods like simultaneous inverse iteration ${ }^{7}$ or condensation methods with general masters ${ }^{4}$ which are usually inferior to the powerful approaches mentioned above could be faster. In particular this could be true in the dynamic analysis of structures since the eigenvalue problem under consideration is the discrete version 
of a system with an infinite number of degrees of freedom which itself is only a model of the real structure. Hence, it does not make sense to compute the eigensolutions with higher accuracy than the modelling error and the discretization error, and therefore the accuracy requirements for approximations to the eigenvalues and eigenvectors are not very high.

Moreover, in a parallel environment a small number of Rayleigh quotient iterations or condensation methods need only little communication whereas in the Lanczos process in every iteration step communication is necessary to perform 2 scalar products, 2 _axpys and to compute $K^{-1} M x$ for some vector $x$, and similar considerations hold for Jacobi-Davidson's method.

In this note we report on numerical experiments computing approximations to some eigenvalues and corresponding eigenvectors of a finite element model of a container ship as they are needed in the analysis of the dynamic response. We considered a parallel implementation of condensation with general masters on two environments: a heterogeneous HP workstation cluster and an HP N-Class parallel computer. We found that it well compares to the Lanczos method implemented in the established package P_ARPACK ${ }^{6}$ if only approximations of low accuracy of a few eigenvalues at the lower end of the spectrum are needed and if reasonable approximations to eigenvectors are available.

Our paper is organized as follows. In Section 2 we briefly sketch condensation with general masters, Section 3 summarizes our parallelization concept using substructuring, and Section 4 describes details of the finite element model under consideration, the accuracy requirements in the response analysis, and the numerical results.

\section{Condensation with general masters}

We consider the general eigenvalue problem (1) where $K \in \mathbb{R}^{(n, n)}$ and $M \in$ $\mathbb{R}^{(n, n)}$ are symmetric and positive definite which are usually the stiffness and the mass matrix of a finite element model of a structure. To deal with the large number of degrees of freedom static condensation is frequently employed to economize the computation of a selected group of eigenvalues and eigenvectors.

To this end the degrees of freedom are decomposed into masters and slaves. After reordering the equations and unknowns problem (1) can be rewritten as

$$
\left(\begin{array}{cc}
K_{m m} & K_{m s} \\
K_{s m} & K_{s s}
\end{array}\right)\left(\begin{array}{c}
x_{m} \\
x_{s}
\end{array}\right)=\lambda\left(\begin{array}{cc}
M_{m m} & M_{m s} \\
M_{s m} & M_{s s}
\end{array}\right)\left(\begin{array}{c}
x_{m} \\
x_{s}
\end{array}\right) .
$$

Neglecting the inertia terms in the second equation, solving for $x_{s}$, and sub-

PelzerVoss: submitted to IC Press on January 29, 2001 
stituting $x_{s}$ into the first equation one obtains the condensed problem

$$
\tilde{K}_{0} x_{m}=\lambda \tilde{M}_{0} x_{m}
$$

where

$$
\begin{gathered}
\tilde{K}_{0}:=K_{m m}-K_{m s} K_{s s}^{-1} K_{s m} \\
\tilde{M}_{0}:=M_{m m}-K_{m s} K_{s s}^{-1} M_{s m}-M_{m s} K_{s s}^{-1} K_{s m}+K_{m s} K_{s s}^{-1} M_{s s} K_{s s}^{-1} K_{s m}
\end{gathered}
$$

Nodal condensation has the disadvantage that it produces accurate results only for a small part of the lower end of the spectrum. The approximation properties can be improved substantially if general masters ${ }^{4}$ are considered. Let $z_{1}, \ldots, z_{m} \in \mathbb{R}^{n}$ be independent vectors, and define $Z:=\left(z_{1}, \ldots, z_{m}\right) \in$ $\mathbb{R}^{(n, m)}$. Then the projected eigenvalue problem

$$
K_{0} x_{m}:=P^{T} K P x_{m}=\lambda P^{T} M P x_{m}=: \lambda M_{0} x_{m},
$$

where

$$
P=K^{-1} Z\left(Z^{T} K^{-1} Z\right)^{-1} Z^{T} Z
$$

is called condensed eigenvalue problem with general masters $z_{1}, \ldots, z_{m}$. It is easily seen that this is exactly the reduced problem of nodal condensation if we choose $z_{1}, \ldots, z_{m}$ as unit vectors corresponding to the master degrees of freedom.

Since $\left(Z^{T} K^{-1} Z\right)^{-1} Z^{T} Z$ is a nonsingular matrix the condensed problem (6) is equivalent to the projection of problem (1) to the space spanned by the columns of $K^{-1} Z$. Hence, condensation is nothing else but one step of simultaneous inverse iteration with initial guess $X=M^{-1} Z \in \mathbb{R}^{(n, m)}$. Therefore, we can expect good approximation properties of condensation if we include general masters $z_{j}=M x_{j}$ into the condensation process where $x_{j}$ are approximate eigenvectors of problem (1) corresponding to the desired eigenvalues. Hence, choosing approximate eigenvectors from previous design steps as general masters should yield reasonable eigenvalue approximations.

In the next section we combine nodal condensation with substructuring to obtain a coarse grained parallelization. To generalize this concept to condensation in the presence of general masters the following result is of great convenience.

Theorem 1 Assume that $Z^{T} Z=I$. Then the projection matrix $Z$ in (7) can be determined from the linear system

$$
\left(\begin{array}{cc}
K & -X \\
-X^{T} & O
\end{array}\right)\left(\begin{array}{c}
P \\
S
\end{array}\right)=\left(\begin{array}{c}
O \\
-I
\end{array}\right) .
$$


Moreover, the condensed stiffness matrix is given by

$$
P^{T} K P=S \text {. }
$$

\section{Parallel Condensation}

For nodal condensation the following strategy yields a coarse grained parallel algorithm ${ }^{8}$ based on the master-worker paradigm. Suppose that the structure under consideration has been decomposed into $r$ substructures and let the masters be chosen as interface degrees of freedom. Assume that the substructures connect to each other through the master variables only. If the slave variables are numbered appropriately, then the stiffness matrix is given by

$$
K=\left(\begin{array}{ccccc}
K_{m m} & K_{m s 1} & K_{m s 2} & \ldots & K_{m s r} \\
K_{s m 1} & K_{s s 1} & 0 & \ldots & 0 \\
K_{s m 2} & 0 & K_{s s 2} & \ldots & 0 \\
\vdots & \vdots & \vdots & \ddots & \vdots \\
K_{s m r} & 0 & 0 & \ldots & K_{s s r}
\end{array}\right)
$$

and the mass matrix $M$ has the same block form.

It is easily seen that in this case the reduced matrices in (3) are given by

$$
\tilde{K}_{0}=K_{m m}-\sum_{j=1}^{r} K_{m m j}:=K_{m m}-\sum_{j=1}^{r} K_{m s j} K_{s s j}^{-1} K_{s m j}
$$

and

$$
\begin{gathered}
\tilde{M}_{0}=M_{m m}-\sum_{j=1}^{r} M_{m m j}, \\
M_{m m j}:=K_{m s j} K_{s s j}^{-1} M_{s m j}+M_{m s j} K_{s s j}^{-1} K_{s m j}-K_{m s j} K_{s s j}^{-1} M_{s s j} K_{s s j}^{-1} K_{s m j} .
\end{gathered}
$$

Hence they can be computed completely in parallel, and the only communication that is needed is one fan-in process to determine the reduced matrices $\tilde{K}_{0}$ and $\tilde{M}_{0}$.

If general masters $z_{1}, \ldots, z_{g}$ are added to the interface masters then according to Theorem 1 the block structure of $K$ in (10) has to be augmented by columns and rows containing the general masters. If the support of each of the general masters is contained in exactly one substructure then the linear 


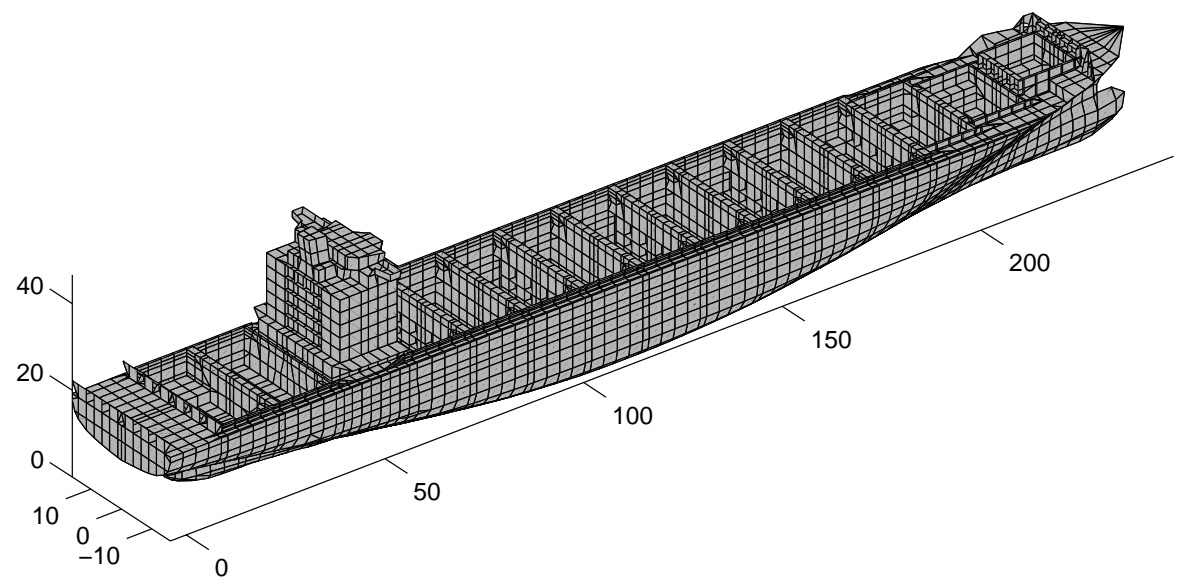

Figure 1. Container ship

system (8) can be solved substructurewise ${ }^{5}$, and the reduced matrices $K_{0}$ and $M_{0}$ again are obtained by a fan-in process.

For general masters having global support as they appear in reanalysis problems we developed a coarse grained parallelization concept which is discussed in detail in a report ${ }^{3}$. In this algorithm the communication consists of two fan-in processes and one broadcast to compute the reduced matrices $K_{0}$ and $M_{0}$.

We implemented the algorithm in Fortran90 using LAPACK 3 and BLAS routines for the linear algebra and MPI 1.05 for message passing. We tested the program on a heterogeneous workstation cluster consisting of (subsets of) one HP J5000, one HP J2240 (each with a double processor), one HP C3000 and five HP 9000, 712/100 connected by fast ethernet, and on an HP N-Class parallel computer with 8 PA $8500 / 440$ Mhz processors organized as one cluster.

\section{Results and Discussion}

To test the performance of the parallel method mentioned in Section 3 we considered the vibrational analysis of a container ship which is shown in Figure 1. Usually in the dynamic analysis of a structure one is interested in the response of the structure at particular points to harmonic excitations of typical forcing frequencies. For instance in the analysis of a ship these are locations 
in the deckshouse where the perception of the crew is particularly strong.

The finite element model of the ship (a complicated 3 dimensional structure) is not determined by a tool like ANSYS or NASTRAN since this would result in a much too large model. Since in-plane displacements of the ship's surface do not influence the displacements in the deckshouse very much it suffices to discretize the surface by linear membrane shell elements with additional bar elements to correct warping, and to model only the main engine and the propeller as three dimensional structures. For the ship under consideration this yields a very coarse model with 19106 elements and 12273 nodes resulting in a discretization with 35262 degrees of freedom.

We consider the structural deformation caused by an harmonic excitation at a frequency of $4 \mathrm{~Hz}$ which is a typical forcing frequency stemming from the engine and the propeller. Since the deformation is small the assumptions of the linear theory apply, and the structural response can be determined by the mode superposition method taking into account eigenfrequencies in the range between 0 and $7.5 \mathrm{~Hz}$ (which corresponds to the 50 smallest eigenvalues for the ship under consideration).

The dynamic behaviour of the ship has to be simulated for different service conditions, i.e. for different velocities and different cargo distributions, and each of these positions yields a specific position of the ship in the water.

When computing the vibrations of a ship embeded in the water the influence of the surrounding fluid on the structure is accounted for in the form of the so-called hydrodynamic masses which have to be added to the masses in the nodal degrees of freedom on the wet surface ${ }^{2}$. Physically, these masses represent the amount of fluid that is accelerated by the vibrating solid.

To summarize, the dynamic analysis of a ship necessitates to solve a couple of sparse generalized eigenvalue problems the mass matrices of which are small modifications of each other. The finite element model is very coarse and therefore the accuracy requirements are very modest. An error of $10 \%$ for the natural frequencies often suffices ${ }^{1}$.

In our experiments we assume that the eigenmodes of the dry ship (i.e. without hydrodynamic masses) corresponding to eigenfrequencies which are less than $7.5 \mathrm{~Hz}$ have been computed in a previous calculation and are known. To determine the modes of a specific wet ship we subdivide the model into 10 substructures (cf. Figure 2 where we attached the number of degrees of freedom to the substructures). Choosing all interface degrees of freedom as masters we obtain a reduced problem of dimension $m=2097$ and the slave subproblems are of dimensions between 1134 and 4792 .

Although the eigenfrequencies of these two models differ quite a bit (the relative differences of the natural frequencies lie between $10 \%$ and $50 \%$ ) the 


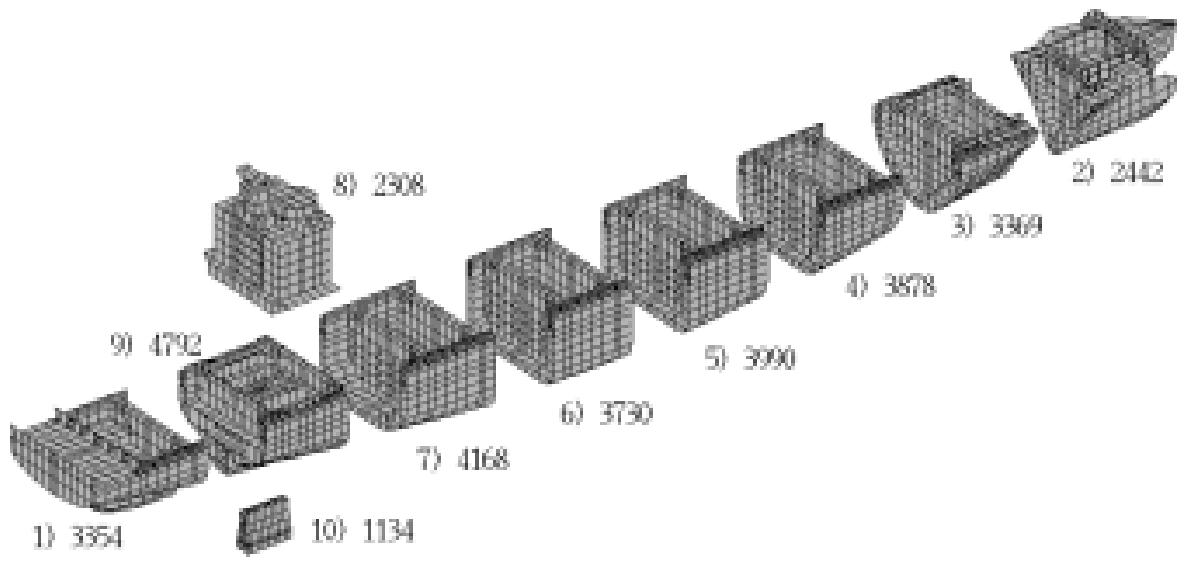

Figure 2. Substructuring of a container ship

approximation properties of the condensation method are enhanced considerably if we add 50 dry modes as general masters to the interface masters when solving the wet model. For nodal condensation only the 15 smallest eigenfrequencies are obtained with a relative error less than $10 \%$ whereas with 50 dry modes as additional general masters the relative error of the 50 smallest eigenfrequencies of the wet model is less than $9.83 \%$.

Next we dropped the interface masters, i.e. we considered only 50 dry modes as general masters in the condensation method. The accuracy decreased only slightly to a maximal relative error of $9.89 \%$ of the eigenfrequency approximations.

We compared the performance of these condensation methods to that of P_ARPACK where we provided vectors $K^{-1} M x$ in the reverse communication interface taking advantage of the substructuring above and the Schur complement and where we tuned the parameters such that we obtained approximations to the 50 smallest natural frequencies with a maximal relative error of about $10 \%$ (actually $12.5 \%$ were arrived). The following table contains the runtimes needed for these 3 methods on the HP N-Class parallel computer and on a network of one HP J5000, one HP J2240, one HP C3000 and 3 HP 9000, 712/100.

\begin{tabular}{lccc} 
& int.face+glob. mast. & glob. mast. & P_ARPACK \\
\hline HP N-Class & 158 & 90 & 249 \\
Workstation cluster & 925 & 273 & 424 \\
Notice that the user can only define processes, and assign these processes
\end{tabular}


to workstations but not to processors. Hence, with a heterogeneous cluster with workstations of different computing speed and different numbers of processors it is not easy to obtain a good load balancing. This is in particular the case since the substructuring can not be changed easily without increasing drastically the number of interface masters and the dimension of the Schur complement, respectively. For the N-Class parallel computer the user defines processes and the local scheduling is organized by the operating system.

\section{Acknowledgements}

Thanks are due to Christian Cabos, Germanischer Lloyd, who provided us with the finite element model of the container ship. The first author gratefully acknowledges financial support of this project by the German Foundation of Research (DFG) within the Graduiertenkolleg "Meerestechnische Konstruktionen".

\section{References}

1. C. Cabos, Private communication 2001

2. C. Cabos and F. Ihlenburg, Vibrational analysis of ships with coupled finite and boundary elements. Report, Germanischer Lloyd 1999. http://www.germanlloyd.org/mba/research/fi/damp/paper.pdf

3. B. Hofferek, A. Pelzer and H. Voss, Global masters in parallel condensation of eigenvalue problems. Report 31, Arbeitsbereich Mathematik, TU Hamburg-Harburg 1999. http://www.tuharburg.de/mat/SCHRIFTEN/Berichte.html

4. W. Mackens and H. Voss, Nonnodal condensation of eigenvalue problems, ZAMM 79, 243 - 255 (1999)

5. W. Mackens and H. Voss, General masters in parallel condensation of eigenvalue problems, Parallel Computing 25, 893 - 903 (1999)

6. K.J. Maschhoff and D.C. Sorensen, P_ARPACK: An efficient portable large scale package for distributed memory parallel architectures. In J. Wasniewski, J. Dongarra, K. Madsen and D. Olesen (eds.), Applied Parallel Computing in Industrial Problems and Optimization, Volume 1184 of Lecture Notes in Computer Science, Springer Verlag, Berlin 1996

7. B.N. Parlett, The Symmetric Eigenvalue Problem, Classics in Applied Mathematics, SIAM, Philadelphia 1998

8. K. Rothe and H. Voss, A fully parallel condensation method for generalized eigenvalue problems on distributed memory computers. Parallel Computing 21, $907-921$ (1995) 